\title{
The properties of jet in luminous blazars under the equipartition condition
}

\author{
Wen $\mathrm{Hu}^{\mathrm{a}, \mathrm{b}, \mathrm{c}}$, Ben-Zhong Dai ${ }^{\mathrm{a}, \mathrm{b}, *}$, Wei Zeng ${ }^{\mathrm{a}, \mathrm{b}}$, Zhong-Hui Fan ${ }^{\mathrm{a}, \mathrm{b}}$, Li Zhang $^{\mathrm{a}, \mathrm{b}}$ \\ ${ }^{a}$ Department of Astronomy, Yunnan University, Kunming 650091, China. \\ ${ }^{b}$ Key Laboratory of Astroparticle Physics, Yunnan Province, Kunming 650091, China. \\ ${ }^{c}$ School of Mathematical Sciences and Physics, Jinggangshan University, Jiangxi Province, Ji'an 343009, \\ China.
}

\begin{abstract}
In this work, we study the physical properties of the high-energy (HE) emission region by modeling the quasi-simultaneous multi-wavelength(MWL) spectral energy distributions (SEDs) of 27 Fermi-LAT detected low-synchrotron-peaked (LSP) blazars. We model the jets MWL SEDs in framework of a well accepted single-zone leptonic model including synchrotron self-Compton and external Compton (EC) processes for the jets in a state of equipartition between particle and magnetic field energy densities. In the model the $\mathrm{GeV} \gamma$-ray spectrum is modeled by a combination of two different external Compton-scattered components: (i) EC scattering of photons coming from disk and broad line region (BLR), and (ii) EC scattering of photons originating from the dust tours (DT) and BLR. We find that the SEDs can be well reproduced by the equipartition model for the most majority of the sources, and the results are in agreement with many recent studies. Our results suggest that the SEDs modelling alone may not provide a significant constraint on the location of the HE emission region if we do not know enough about the physical properties of the external environment.
\end{abstract}

Keywords: galaxies: active; radiation mechanisms: non-thermal; quasars: general; $\gamma$ rays: observations

\section{Introduction}

Blazars are a subclass of radio-loud active galactic nuclei (AGNs), and typically exhibit substantial variability across the electromagnetic spectrum from radio to $\gamma$ rays originating in a relativistic jet pointing toward us (Urry et al., 1995). Traditionally, blazars are comprised of flat spectrum radio quassars (FSRQs) and BL Lac objects. In particular, FSRQs are the most interesting $\gamma$ ray objects for studying the effects of jet acceleration as well as its interaction with the surrounding ambient medium. FSRQs show strong broad prominent emission lines and are commonly associated with high luminosity radio galaxies (Fanaroff-Riley type II [FR II]; Fanaroff \& Riley (1974))

\footnotetext{
${ }^{*}$ Corresponding author

Email address: bzhdai@ynu. edu.cn (Ben-Zhong Dai )
}

Preprint submitted to Elsevier

October 6, 2016

C 2016. This manuscript version is made available under the Elsevier user license http://www.elsevier.com/open-access/userlicense/1.0/ 
whereas BL Lacs have weak or absent emission lines and are associated with the lower luminosity Fanaroff-Riley type I [FR I]. The broadband continuum spectra of blazars are dominated by non-thermal emission and consist of at least two clearly distinct broad spectral components. In FSRQs the first component is usually in the infrared regime, while for BL Lacs it is between infrared and hard X-rays. It is commonly accepted that the first component is usually interpreted as synchrotron emission of electrons while the second component is believed to originate from inverse-Compton (IC) up-scattering off synchrotron (SSC; Maraschi et al., 1992; Bloom \& Marscher, 1996) or external photons from the disk (ECD; Dermer \& Schlickeiser, 1993; Dermer et al., 2009), or radiation reprocessed and scattered in nearby clouds (ECC; Sikora et al., 1994; Hu et al., 2015), and/or infra-red (IR) photons from the dusty obscuring torus (ECT; Blazejowski et al., 2000).

Therefore, the simultaneous and/or quasi-simultaneous MWL SEDs are a basic tool to study the underlying mechanisms of energy dissipation (Vlahakis et al., 2004; Marscher et al., 2008), and the physics properties of blazars, such as magnetic field strength, the Doppler factor, the and dimension and location of HE emission region. Based on the simultaneous and/or quasi-simultaneous MWL SEDs, many authors have studied the relevant properties of blazars in the framework of the leptonic model (e.g., Ghisellini et al., 2010, 2011; Ghisellini \& Tavecchio , 2014; Cao et al., 2011; Yan et al., 2012; Kang et al, 2014). These works are based on the assumption of a spherical geometry model for the BLR and/or DT. However, some of the basic facts concerning the BLR in particular are still under debate. For instance, it is unclear whether the BLR has a spherical or flat geometry (Jarvis \& McLure, 2006; Labita et al., 2006; Decarli et al., 2008, 2011). The knowledge of the BLR geometry of AGN plays a crucial role in estimating sites of the observed radiation spectra and black hole masses from the widths of the broad emission lines (Peterson et al., 1993, 2004, and references therein). In addition, there is some observational evidence that BLR material may be present downstream of the radio core located at parsec scales from the central black hole (León-Tavares et al., 2010, 2013). Moreover, the location of the blazar $\gamma$-ray emission site is regarded as one of the central questions debated in the Fermi era. To date, it does not make clear whether the $\gamma$-ray emission is produced inside the sub-pc size BLR or further out at scales of about 1 - few pc where the IR photon field of the DT dominates over that of the UV field of the BLR (e.g., Tavecchio et al., 2010; Marscher et al., 2010; Agudo et al., 2011; Liu et al., 2011; Dotson et al., 2012; Harris et al., 2012; Orienti et al., 2013; Kang et al, 2014; Nalewajko et al., 2014a).

As mentioned above, one should take into account such uncertainties as location of blzaar zone and geometry of the external radiation fields. The paper is structured as follows: Section 2 provides a description of the SEDs modeling for a sample of Fermi-detected blazars. Section 3 describe the sample, and Section 4 show the results of the SEDs modeling. The summary and discussions are presented in Section 5. Throughout the work, we adopted a standard cosmology with parameters $H_{0}=70 \mathrm{~km} / \mathrm{s} / \mathrm{Mpc}, \Omega_{m}=0.3$, and $\Omega_{\Lambda}=0.7$. 


\section{The modeling procedure of the observed SEDs for Fermi-detected blazars}

In a relativistic jet, an emission region responsible for the high energy $\gamma$ ray emission propagates with relativistic speed $\beta_{b}=\left(1-1 / \Gamma_{b}\right)^{1 / 2}$ outward along the jet, which is directed at an angle $\theta_{o b s}=\cos ^{-1} \mu_{o b s}$ with respect to the line of sight. Thus, the observed photons are beamed and Doppler-boosted towards the observer, and the Doppler boosting of emission is determined by the Doppler factor $\delta_{b}=\left[\Gamma_{b}\left(1-\beta_{b} \mu_{o b s}\right)\right]^{-1}$. The emission region is modeled as a spherical magnetized plasma cloud of radii $R_{b}^{\prime}$ which consists of a randomly oriented magnetic field $B^{\prime}$ and a population of isotropic relativistic particles. The electron energy distribution (EED) reconstructed from the observed SEDs of luminous blazars is best approximated by a broken power law form with normalization $k_{e}^{\prime}$ between $\gamma_{\min }^{\prime}$ and $\gamma_{\max }^{\prime}$, with slopes $p_{1}$ and $p_{2}$ below and above the break at $\gamma_{b r}^{\prime}$, and is given by,

$$
n_{e}^{\prime}\left(\gamma^{\prime}\right)=k_{e}^{\prime}\left\{\begin{array}{ll}
\left(\gamma^{\prime} / \gamma_{b r}^{\prime}\right)^{-p_{1}} & \text { for } \gamma_{\min }^{\prime} \leq \gamma^{\prime} \leq \gamma_{b r}^{\prime} \\
\left(\gamma^{\prime} / \gamma_{b r}^{\prime}\right)^{-p_{2}} & \text { for } \gamma_{b r}^{\prime}<\gamma^{\prime} \leq \gamma_{\max }^{\prime}
\end{array} .\right.
$$

In the HE emission region, the electrons emit via synchrotron and IC mechanisms, which are are well known as the basic radiative processes in blazar jets (e.g., Ludovic \& Gilles, 2004; Crusius \& Schlickeiser, 1986; Blumenthal \& Gould, 1970; Dermer \& Schlickeiser, 2002; Dermer et al., 2009; Tang et al., 2010).

In order to deduce model parameters including $\delta_{b}, B^{\prime}, R_{b}^{\prime}, \gamma_{b r}^{\prime}$, and $k_{e}^{\prime}$, we infer the analytic relations between the relevant physical parameters of the considered model and the observable quantities provided by the observed SEDs including the peak synchrotron frequency $\nu_{s, p k}$, its luminosity $\nu_{s y n}^{p k} L_{s y n}^{p k}$, the SSC dominance factor $\xi_{s}$ (or the peak SSC luminosity $\left.\nu_{s s c}^{p k} L_{s s c}^{p k}\right)$ and the peak SSC frequency $\left(\nu_{c, p k}\right)$. The analytic results are described in Appendix A, where we also show how the other parameters characterising the non-thermal EED are constrained by the observed SEDs. In our fitting procedure, a visual inspection of the fitted SEDs is used, and the parameters are estimated in such a way as to best reproduce the observed MWL SEDs of all LSP blazars, and we would like to do this by varying the least number of parameters possible between objects. Finally, the model parameters are used to calcuate the synchrotron, synchrotron Self-Compton and external Compton-scattered fluxes, and the formula for calculating the SEDs are given in Finke et al. (2008) and Dermer et al. (2009). In our numerical calculation the higher-order SSC components and synchrotron self-absorption (SSA) are considered consistently. In particular, we assume that a equipartition relation holds between the magnetic-field and relativistic electron energy densities, i.e., $\xi_{e}=1$, which is supported by previous works (e.g., Ghisellini et al., 2010; Yan et al., 2012; Cao \& Wang, 2013) and numerical modeling of the observed SEDs (e.g., Böttcher \& Chiang , 2002; Schlickeiser \& Lerche, 2007, 2008). The principle of equipartition provides a minimum power solution for blazars jet emissions, when the synchrotron peak dominated (Dermer \& Atoyan, 2004; Finke et al., 2008; Dermer et al., 2014). 


\subsection{Calculations of External Compton Scattering}

In the case of isotropic soft photon field, the Compton spectral flux is calculated by

$$
\epsilon F_{\epsilon}^{i s o}=\frac{c V_{b}^{\prime} \epsilon_{s}^{2} \delta_{b}^{3}}{4 \pi d_{L}^{2}} \int_{0}^{\infty} d \epsilon_{*} \frac{u_{*}\left(\epsilon_{*}\right)}{\epsilon_{*}} \int_{\gamma_{\min }}^{\gamma_{\max }} d \gamma n_{e}^{\prime}\left(\gamma / \delta_{b}\right) P_{i s o}\left(\epsilon_{s}, \gamma, \epsilon_{*}\right)
$$

where $\epsilon=(1+z) \epsilon_{s}, V_{b}^{\prime}=4 \pi R_{b}^{\prime 3} / 3$ is the comoving volume of the blob, and the differential scattering cross section is $P_{\text {iso }}\left(\epsilon_{s}, \gamma, \epsilon_{*}\right)=\frac{3 \sigma_{T}}{4 \epsilon_{*} \gamma^{2}}\left[2 x \ln x+x+1-2 x^{2}+\right.$ $\left.\frac{(4 \epsilon \gamma x)^{2}}{2(1+4 \epsilon \gamma x)}(1-x)\right] H\left(x ; \frac{1}{4 \gamma^{2}}, 1\right)$, with $x=\frac{\epsilon_{s}}{4 \epsilon_{*} \gamma\left(\gamma-\epsilon_{s}\right)}$. The lower limit $\gamma_{\min }$ and the upper limit $\gamma_{\max }$ implied by the kinematic limits on $x$ are given by

$\gamma_{\min }=\frac{1}{2} \epsilon_{s}\left(1+\sqrt{1+\frac{1}{\epsilon_{*} \epsilon_{s}}}\right)$, and, $\gamma_{\max }=\frac{\epsilon_{*} \epsilon_{s}}{\epsilon_{*}-\epsilon_{s}} H\left(\epsilon_{*}-\epsilon_{s}\right)+\delta_{b} \gamma_{\max }^{\prime} H\left(\epsilon_{s}-\epsilon_{*}\right)$,

where the Heaviside function $H(x)=0$ for $x<0$ and $H(x)=1$ for $x \geq 0$. The specific energy density $u_{*}\left(\epsilon_{*}\right)$ is expressed as a graybody, and is given by Planck's law:

$$
u_{*}\left(\epsilon_{*}\right)=\varsigma \frac{8 \pi m_{e} c^{2}}{\lambda_{c}^{3}} \frac{\epsilon_{*}^{3}}{\exp \left(\epsilon_{*} / \Theta\right)-1}=\frac{15 u_{*}}{(\pi \Theta)^{4}} \frac{\epsilon_{*}^{3}}{\exp \left(\epsilon_{*} / \Theta\right)-1}
$$

where $\lambda_{c}=h / m_{e} c=2.43 \times 10^{-10} \mathrm{~cm}$ is the electron Compton wavelength, $m_{e}$ is the rest mass of electron, $c$ is the speed of light, and $h$ is Planck's constant. Here, the factor $\varsigma \equiv \frac{u_{*}}{a T_{*}^{4}}$, where $u_{*}$ is the energy density of the external radiation field with a dimensionless temperature $\Theta \equiv k_{B} T_{*} / m_{e} c^{2}$, and the radiation constant can be defined in terms of the Boltzmann's constant $k_{B}$ as $a \equiv \frac{8 \pi^{5} k_{B}^{4}}{15 h^{3} c^{3}}=7.57 \times 10^{-15} \mathrm{ergs} / \mathrm{cm}^{3} / \mathrm{K}^{4}$. Thus, a blackbody is a graybody with $\varsigma=1$.

In the case of anisotropic soft photon field from an accretion disk, the $\epsilon F_{\epsilon}$ flux is given by

$$
\epsilon F_{\epsilon}^{\text {aniso }}=\frac{c V_{b}^{\prime} \epsilon_{s}^{2} \delta_{b}^{3}}{4 \pi d_{L}^{2}} \oint d \Omega_{*} \int_{0}^{\infty} d \epsilon_{*} \frac{u_{*}\left(\epsilon_{*}, \Omega_{*}\right)}{\epsilon_{*}} \int_{\gamma_{\min }}^{\infty} d \gamma n_{e}^{\prime}\left(\gamma / \delta_{b}\right) P_{h o}\left(\epsilon_{s}, \gamma, \bar{\epsilon}\right)
$$

where the differential scattering cross section is $P_{h o}\left(\epsilon_{s}, \gamma, \bar{\epsilon}\right)=\frac{3 \sigma_{T}}{8 \gamma^{2} \epsilon_{*}}\left[y+y^{-1}-\right.$ $\left.\frac{2 \epsilon_{s}}{\gamma \bar{\epsilon} y}+\left(\frac{\epsilon_{s}}{\gamma \bar{\epsilon} y}\right)^{2}\right] H\left(\epsilon_{s} ; \frac{\bar{\epsilon}}{2 \gamma}, \frac{2 \gamma \bar{\epsilon}}{1+2 \bar{\epsilon}}\right)$, with $y \equiv 1-\frac{\epsilon_{s}}{\gamma}$. Here the invariant energy $\bar{\epsilon}=$ $\gamma \epsilon(1-\beta \cos \psi) \simeq \gamma \epsilon\left(1-\mu \mu_{o b s}\right)$, and the lower limit on the electron Lorentz factor is $\gamma_{\text {min }}=\frac{\epsilon_{s}}{2}\left(1+\sqrt{1+\frac{2}{\epsilon_{*} \epsilon_{s}(1-\cos \psi)}}\right)$.

\subsection{The external photon field around the emission region}

Here, the $\mathrm{GeV} \gamma$ ray spectrum is modeled by a superposition of two different external Compton-scattered components (Finke \& Dermer, 2010; Cerruti et al., 2013; Dermer et al., 2014). There are two alternative scenarios proposed. In scenario (i), we focus our attention on the standard scenario where the dense radiation field directed from the accretion disk and from the BLR are important (called Model A). Consequently, the 
location of the emission region can be obtained, because the importance of Comptonscattered disk radiation depends on the distance of the blazar emission region from the disk. In scenario (ii), the $\gamma$-ray spectrum is produced by a combination of external Compton-scattered BLR (ECC) and DT (ECT) radiation, while the direct accretion disk photons are negligible (called Model B). In the case, the location of the emission region from the central engine can be estimated as $Z_{l o c} \simeq 0.5\left(L_{d} R_{I R}^{2} / L_{I R}\right)^{1 / 2} \simeq$ $9 L_{d, 46} / T_{250}^{-4} \mathrm{pc}$, where $L_{I R}=4 \pi R_{I R}^{2} \sigma_{S B} T_{D T}^{4}$ is the total IR luminosity, and the extension of the dust could be roughly estimated as $R_{I R} \simeq \Gamma_{b}^{2} c t_{\text {var }} /(1+z)$ (Kushwaha et al., 2013).

For the emission spectrum of an accretion disk surrounding the supermassive $\mathrm{BH}$, we consider the cooling, optically thick blackbody solution of Shakura \& Sunyaev (1973). The energy density of the Shakura-Sunyaev accretion-disk model along the jet axis is expressed with a Planckian distribution (Dermer \& Schlickeiser, 2002; Dermer et al., 2009). The effective temperature at radius $R$ of the accretion disk is $T(R)=$ $\left[3 R_{g} L_{d} \varphi(R) / 8 \pi \eta \sigma_{S B} R^{3}\right]^{1 / 4}$, where $\varphi(R)=\left[1-\beta_{i}\left(R_{i} / R\right)^{1 / 2}\right]$. For the Schwarzschild $\mathrm{BH}, \beta_{i} \simeq 1, R_{i}=6 R_{g}$ is the innermost stable orbit and $R_{g} \simeq 1.48 \times 10^{13} M_{8}$ is the gravitation radius. $\eta=1 / 12$ is the efficiency to transform accreted matter into escaping radiation energy. The luminosity of the accretion disk is given through

$$
\epsilon L_{d i s k}(\epsilon)=4 \pi N_{d} \int_{R_{i}}^{R_{o}} R d R \frac{8 \pi m_{e} c^{3}}{\lambda_{c}^{3}} \frac{\epsilon_{*}^{4}}{\exp \left(\epsilon_{*} / \Theta(R)\right)-1},
$$

where $\epsilon_{*}=(1+z) \epsilon$. Here, the observed SED from the accretion disc is normalized to $L_{d}$, and the normalization factor is $N_{d}=L_{d} / 16 \pi \int_{R_{i}}^{R_{o}} \sigma_{S B} T^{4}(R) R d R$, where the Stefan-Boltzmann constant $\sigma_{S B}$ is related to the radiation constant according to the expression $a=4 \sigma_{S B} / c$. The peak is determined by $T_{\max }$. By assuming that most of luminosity is produced within $20 R_{g}, T_{\max }$ can be estimated by (Ghisellini, 2013)

$$
k_{B} T_{\max } \simeq\left(L_{d} / 1600 \pi R_{g}^{2} \sigma_{S B}\right)^{1 / 4} \simeq 10.3\left(\ell_{e d d} / M_{8}\right)^{1 / 4} \mathrm{eV}
$$

where the Eddington ratio is defined as $\ell_{e d d}=L_{d} / L_{e d d}$, and the Eddington luminosity is $L_{e d d}=4 \pi G M_{B H} m_{p} c / \sigma_{T} \simeq 1.26 \times 10^{46} M_{8} \mathrm{erg} / \mathrm{s}$. From the observed $\gamma$-ray flux, the energy density of accretion disk photon field required to reproduce the SEDs can be derived $U_{d}\left(z_{l o c}\right)=\frac{2 N_{d}}{c} \int_{R_{i}}^{R_{o}} \frac{z_{l o c} R \sigma_{S B} T(R)^{4} d R}{\left(z_{l o c}^{2}+R^{2}\right)^{3 / 2}}$. Thus, the parameter $M_{B H}=M_{8} 10^{8} M_{\odot}$ and $L_{d} / L_{e d d}$ are estimated by SEDs modeling of the optical/UV data with a standard Shakura-Sunyaev disk model (Celotti \& Ghisellini, 2008; Ghisellini et al., 2010; Castignani et al., 2013; Castignani \& De Zotti, 2015).

For the BLR and DT, the producing radiation are independent of the detailed geometry which is still under debate along with its extension. For simplicity, the external radiation originating in the BLR and in the DT are assumed to be locally isotropic, and both are modeled as a black-body spectrum (Tavecchio \& Ghisellini, 2008; Nenkova et al., 2008; Landt et al., 2010; Malmrose et al., 2011). The BLR and DT are assumed to be located at $R_{B L R} \simeq 10^{17} L_{d, 45}^{1 / 2} \mathrm{~cm}\left(\right.$ or $\left.0.1 L_{d, 46}^{1 / 2} p c\right)$ and $R_{D T} \simeq 2.5 \times 10^{18} L_{d, 45}^{1 / 2} \mathrm{~cm}$ (or $2.5 L_{d, 46}^{1 / 2} p c$ ), respectively. The critical difference between the BLR and the DT is the energy of the seed photons: the energy distribution of the BLR peaks at UV energies while in the DT it peaks at IR energies. In this work, the temperature of the BLR 
is assumed to be a fraction $\zeta_{B L R} \leq 1$ of the maximum temperature of the disk, i.e., $T_{B L R}=\zeta_{B L R} T_{\max }$. A particular DT is characterised by $T_{D T}=\zeta_{D T}\left[U_{D T} / a\right]^{1 / 4}$ in which $U_{D T}$ is the energy density intercepted and reprocessed by the DT. We set $\zeta_{B L R}=1$ and $\zeta_{D T}=1$ (Cleary et al., 2007; Ghisellini \& Tavecchio, 2009). Here, the energy density $U_{e x t}$ of external radiation fields can be inferred by the quasisimultaneous MWL SEDs modeling with a standard leptonic model.

\section{The Sample}

In the work, the collected MWL observed SEDs are taken from Abdo et al. (2010), which have been accumulated over one year of Fermi operations, are therefore quasisimultaneous but not really simultaneous. The simultaneous MWL SEDs for 105 blazars were constructed by (Giommi et al., 2012), where the simultaneous Fermi data are poorly sampled for most sources. We selected 27 LSP blazars with good MWL SED coverage, in particular, at $\gamma$-ray band. The subset of blazars contains 25 FSRQs and 2 low-frequency BL Lac objects, which may be essentially similar and their different observed properties could result from the different accretion rates feeding their central engines (Ghisellini \& Tavecchio, 2008; Ghisellini et al., 2009; Elitzur, 2014). The features of line-less BL Lac objects may be completely hidden by the boosted non-thermal continuum from the jets, and/or the accretion rate is below a critical value. Many authors indicated that the pure SSC model may fails to fit the SEDs of LBLs (e.g., Kushwaha et al., 2013; Böttcher et al., 2013; Yan et al., 2014; Sanchez et al., 2015). Additionally, the archival data taken from the NASA Extragalactic Data base are used to disentangle the non-thermal beamed jet emission from thermal radiation produced by the accretion disc.

\section{The Results of SEDs modelling}

The overall spectrum of 27 LSP blazars are shown in Fig. B.5, in which the results of Model A are shown in left panel and Model B in right panel for each source. In both case, we are able to produce the spectral breaks with a superposition of two different $\gamma$-ray emission components. The input and output parameters are summarized in Table 1 and Table 2, respectively. Notice that the changed parameter values in Model A and in Model B are separated by the symbol ' $/$. The locations estimated from the covering factor are listed in column 15 of the Table 1. In Table 2 we also outlined the minimum variability time-scales, which are derived from the causality argument and are consistent with the day-scale variability seen in most Fermi-detected FSRQs. It is worth note that the collected SEDs represent an average emission state, and then the derived parameters only represent the average properties of the emission region. We do not focus on account for the rapid variability of gamma ray emission as measured by the Fermi-LAT. 


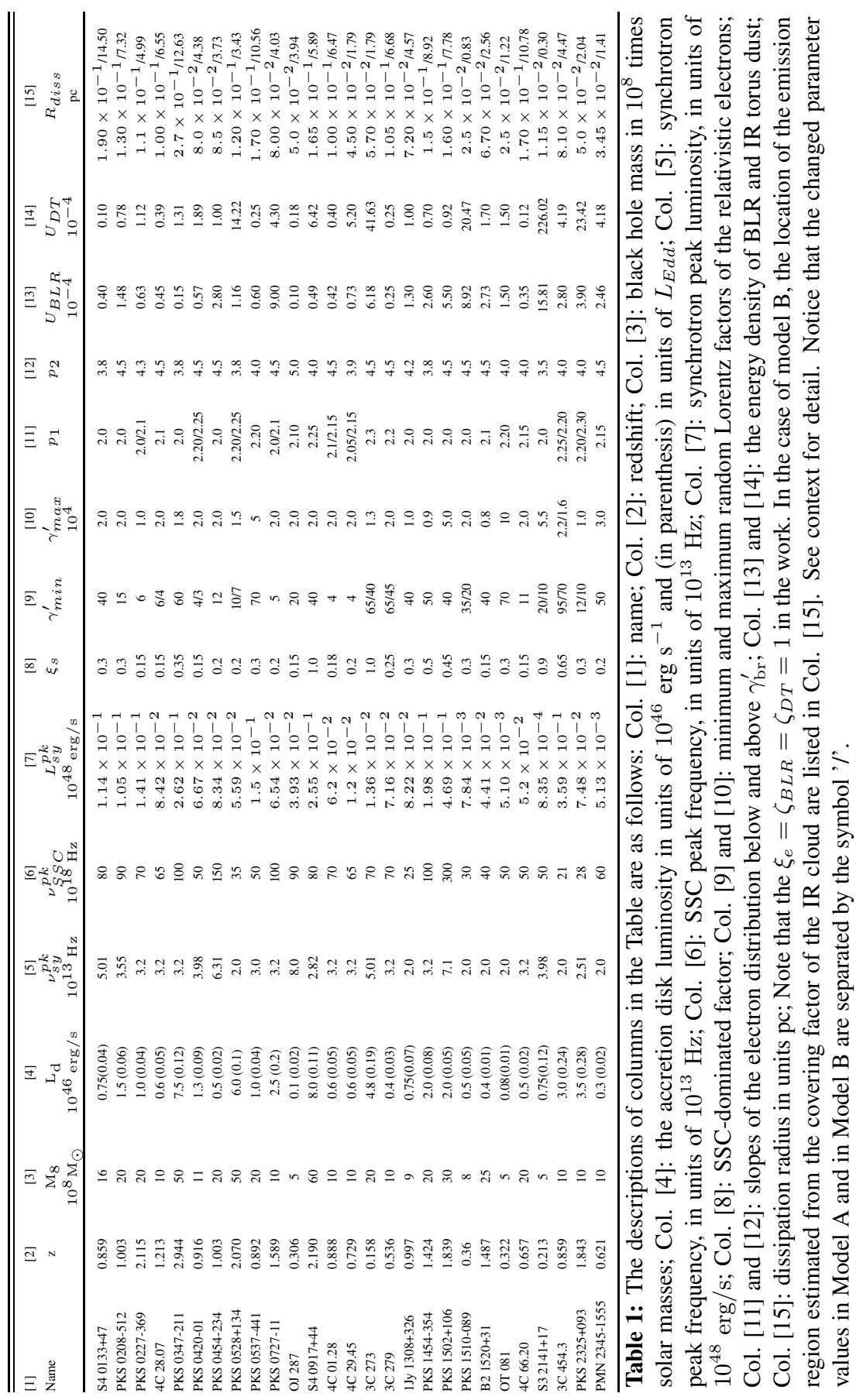



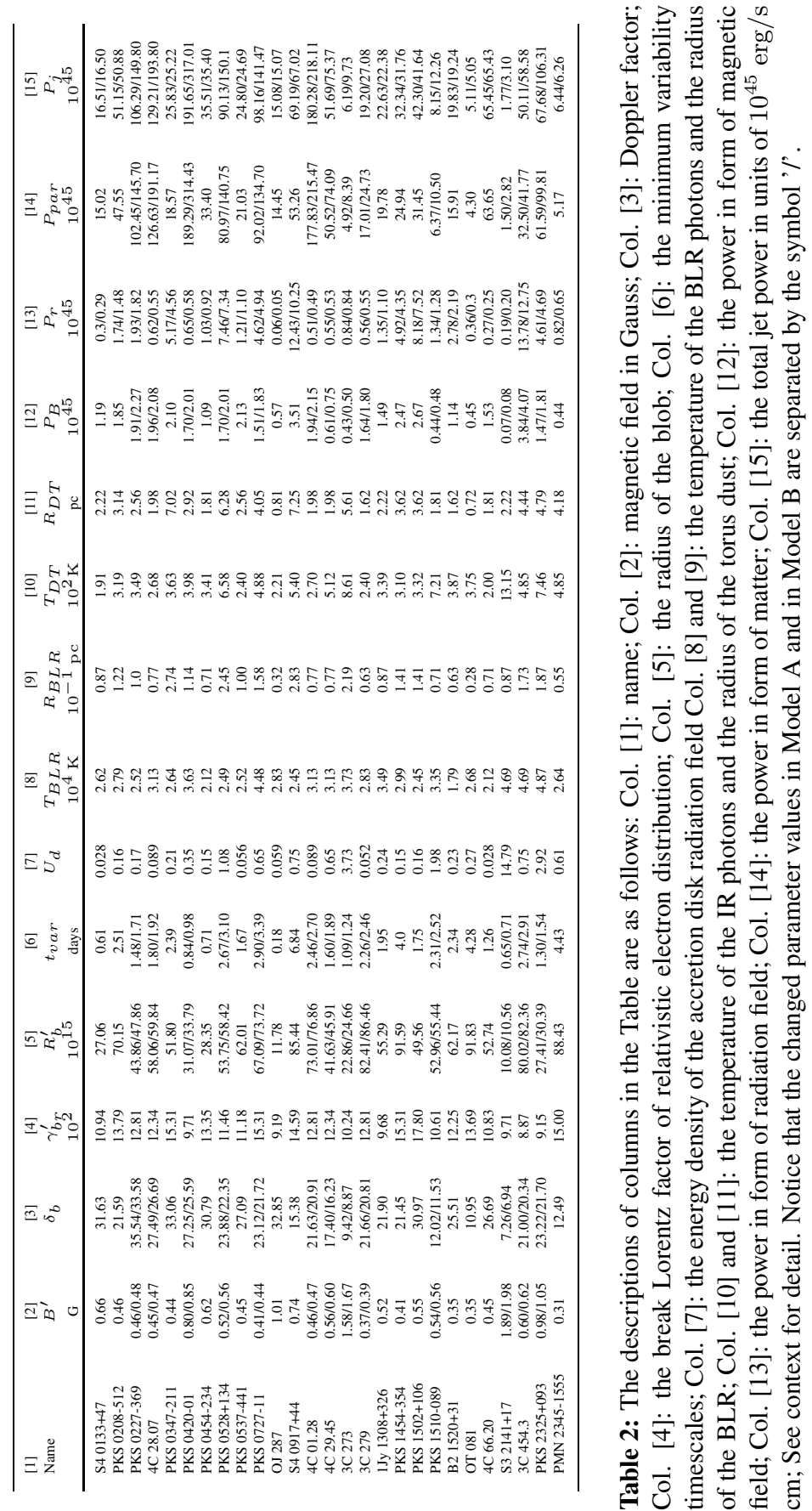
In Fig.1, we compare peak luminosities, peak frequencies and Compton dominance between ours and those obtained from Abdo et al. (2010). The top left panel is for the ratio of synchrotron peak frequency $\nu_{s y}^{p k} / \nu_{s y}^{p k, *}$ comparison, top right for the ratio of synchrotron peak luminosity $L_{s y}^{p k} / L_{s y}^{p k, *}$ comparison, bottom left for the ratio of IC peak frequency $\nu_{C}^{p k} / \nu_{C}^{p k, *}$ comparison, and bottom right for the ratio of Compton dominance $\xi_{C} / \xi_{C}^{*}$ comparison. Here, quantities without asterisks denote values estimated from our physical model, while quantities with asterisks denote values derived from phenomenological approaches. We calculated the $L_{p k}^{s y, *}=4 \pi d_{L}^{2} \nu_{p k}^{s y} F\left(\nu_{p k}^{s y}\right)$, and have corrected $\nu_{p k}^{s y, *}$ and $\nu_{p k}^{C, *}$ to how they would appear in the rest frames of the sources for effective comparison(i.e., $k$-corrected them). The results show that differences between $\nu_{s y}^{p k}$ and $\nu_{s y}^{p k, *}$ decrease as $\nu_{s y}^{p k, *}$ increases, subsequently increase with $\nu_{s y}^{p k, *}$, and the values of $\nu_{s y}^{p k}$ is larger than ones of $\nu_{s y}^{p k, *}$ for most of sources. The $\nu_{C}^{p k, *}-\nu_{C}^{p k} / \nu_{C}^{p k, *}$ relation has quite similar with the $\nu_{s y}^{p k, *}-\nu_{s y}^{p k} / \nu_{s y}^{p k, *}$ relation in the $\log$-log representation, but the values of $\nu_{C}^{p k}$ is smaller than ones of $\nu_{C}^{p k, *}$ for majority of sources. On the other hand, the peak synchrotron luminosities cluster closely around the line of $L_{s y}^{p k}=L_{s y}^{p k, *}$, and are concentrated with a area delimited by the gray line $L_{s y}^{p k}=10^{ \pm 0.5} L_{s y}^{p k, *}$. In the bottom right panel we see $\xi_{C} \gtrsim \xi_{C}^{*}$ for a large fraction of our sources. The majority of the points fall within a area delimited by the gray line $\xi_{C}=10^{ \pm 0.5} \xi_{C}^{*}$. Here, the peak Compton frequencies were estimated via $\nu_{p k}^{C}=(4 / 3) \nu_{D T} \gamma_{b r}^{2} \delta_{b}^{2}$, and the Compton dominance were estimated via $\xi_{C}=\Gamma_{b}^{2} U_{D T} / U_{B}^{\prime}$. In fact, an accurate numerical results indicated that $\nu_{s y}^{p k}, \nu_{C}^{p k}, L_{s y}^{p k}$ are respectively a factor of $\sim 0.75-1.2,1.0-2.0$ and $0.6-0.75$ in our simplified analytic estimates for them, while $\xi_{C}$ is quite accurate when IC scattering of the DT radiation happens in the Thomson regime. Thus, the difference between ours and theirs should be smaller, and our results should be reasonable. The difference may be due to that the radio radiation was treated as an upper limit, and $\nu_{C}^{p k}$ was primarily determined by the $\gamma$-ray data in our work.

In Fig. 2 we report the maximum energy of $\gamma$ ray photons for all sources in our sample, and plot contours of the logarithm of the optical depth to pair production for $\gamma$ rays interacting with EBL. The optical depth for the $\gamma \gamma$ attenuation is calculated by using the formula presented in (Nikishov, 1961; Gould \& Schréder, 1967). Here, the EBL model given by Finke et al. (2010b) was used to calculate the opacity which is modified by multiplying a factor of 0.86 (Ackermann et al., 2012). It implies that the $\gamma \gamma$ attenuation could be neglected, except for PKS 1502. On the other hand, the gamma-gamma opacity in BLR radiation field can also be neglected due to a low energy density of BLR radiation field, while the absorption coming from the accretion disk is only important at $\nu>10^{25} \mathrm{~Hz}$ (Dermer et al., 2009; Yan et al., 2013; Hu et al., 2015).

\section{Summary and Discussion}

\subsection{The derived physical parameters from SEDs modeling}

In the section, we show the derived physical parameters, including $\delta_{b}, B^{\prime}, \gamma_{\min }^{\prime}$, $\gamma_{b r}^{\prime}, \gamma_{\max }^{\prime}$ and the jets power, and compare with that from other authors. We show the distribution of the magnetic field in the upper left panel and the Doppler factor in upper 

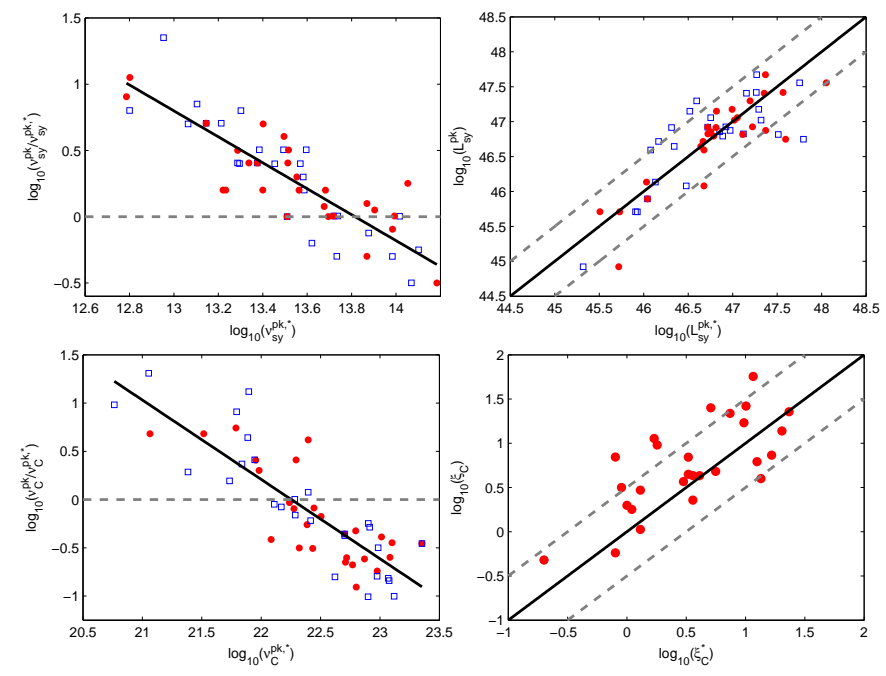

Figure 1: The values of spectral parameters estimated from our physical model $\left(\nu_{s y}^{p k}, L_{s y}^{p k}, \nu_{C}^{p k}, \xi_{C}\right)$ versus those derived from phenomenological approaches $\left(\nu_{s y}^{p k, *}, L_{s y}^{p k, *}, \nu_{C}^{p k, *}, \xi_{C}^{*}\right)$. Red solid circles are directly estimated from SED with threedegree polynomials, while blue open boxes are estimated from an empirical relation between radio flux density, synchrotron peak frequency, and synchrotron peak flux. In the top left and bottom left panels, the black lines represent the best linear fits.

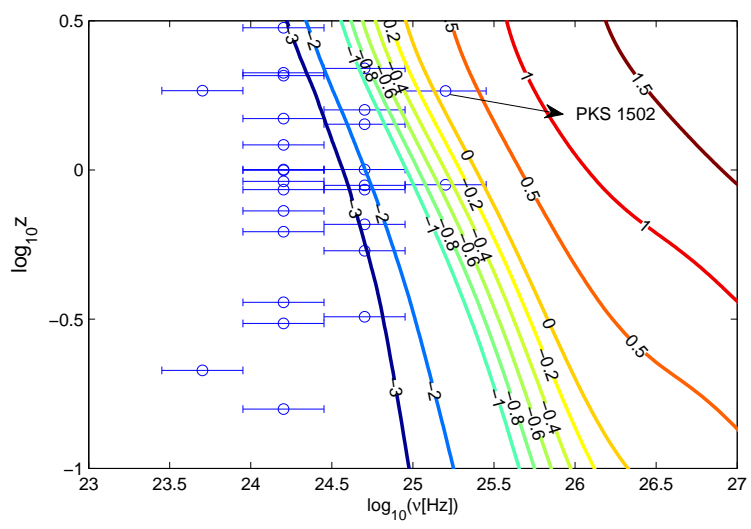

Figure 2: Contour plot of the log of the $\gamma \gamma$ optical depth for a $\gamma$-ray photon interacting with the EBL. The different line corresponds to the different log of the $\gamma \gamma$ optical depth, which are labelled in the figure. Opened circles represent the maximum $\gamma$-ray energy for all sources in our sample. 

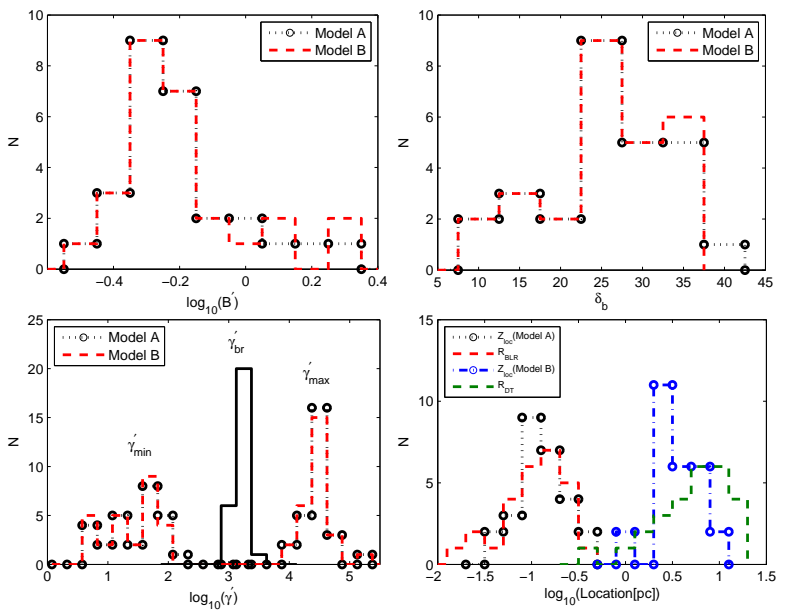

Figure 3: Distributions of derived parameters of magnetic field $\left(B^{\prime}\right)$, Doppler factor $\left(\delta_{b}\right)$, minimum Lorentz factor $\left(\gamma_{\text {min }}^{\prime}\right)$, break Lorentz factor $\left(\gamma_{b r}^{\prime}\right)$ and the maximum Lorentz factor $\left(\gamma_{\text {max }}^{\prime}\right)$ of the distribution of electrons. The distributions of location of the emission region and radius of the BLR and DT are shown in right bottom panel.

right panel of Fig. 3. The values of the Doppler factor are ranging from 7 to 36 are reconciled with those inferred by other authors using different method (Jorstad et al., 2005; Savolainen et al., 2010). The values of magnetic field range between $0.3-2.0$, which are smaller than the results derived by Ghisellini et al. (2010), but larger than the results of Yan et al. (2012). Our results are in accordance with the results of Cao \& Wang (2013); Kang et al (2014). It is obvious that the emitting region is more compact, and has a smaller Doppler factor and a greater magnetic field when it is located closer to the central BH.

In the lower left of Fig. 3, the distributions of $\gamma_{\text {min }}^{\prime}, \gamma_{b r}^{\prime}$ and $\gamma_{\text {max }}^{\prime}$ are presented. For the relativistic electron's distribution, we find that the values of the $\gamma_{\min }^{\prime}$ range between $4-95$, which plays a key role in jet power estimation and are consistent with the results derived by Ghisellini et al. (2010) and Kang et al (2014). Our results indicate that the values of $\gamma_{b r}^{\prime}$ cluster around $\sim 10^{3}$, and $\gamma_{\max }^{\prime}$ cluster around $\sim 2 \times 10^{4}$.

The above compare show that our results may be reasonable and acceptable. Both of our models can reproduce well the quasi-simultaneous emission from optical - X-ray to $\mathrm{GeV}$ band of most sources. The low energy component of the SEDs is ascribed to the synchrotron radiation of the relativistic electrons. It should be noted that the thermal emissions of PMN 2345-1555, 3C 273 and S3 2141+17 are so significant that the optical-UV emission are purely from the thermal emission. The EC process dominates in the X-ray band for six sources, i.e. PKS 0227, 4C 29.45, PKS 0727, PKS 0420, PKS 2325, PKS 0528, while the X-ray emission of 17 out of 27 sources is dominated by the SSC process. For some cases (S3 2141, 4C 01.28, 4C 28.07 and PKS 1510), the $\mathrm{X}$-ray emission originating from SSC and from EC component are comparable. For 

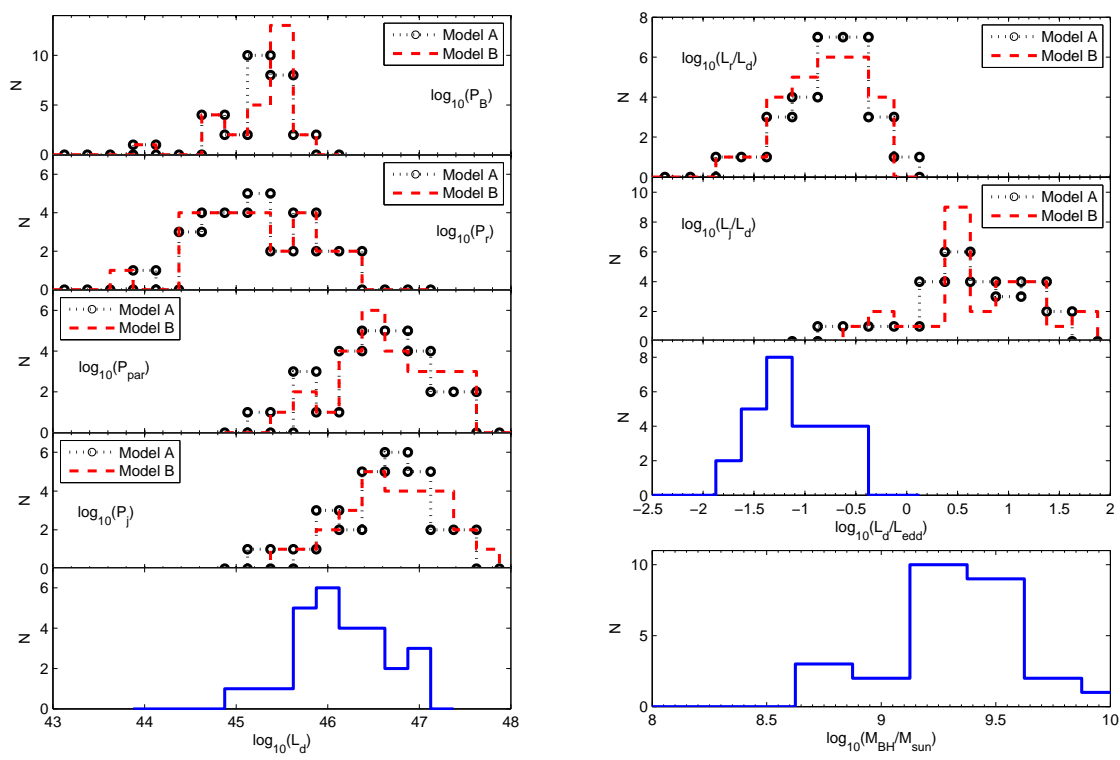

Figure 4: Left panel: distributions of the jet power in the form of magnetic field $\left(P_{B}\right)$, produced radiation $\left(P_{r}\right)$, particles $\left(P_{\text {par }}\right.$, including relativistic electrons and bulk kinetic power of cold protons) and the total jet power $\left(P_{j}\right)$; The bottom panel shows the distribution of the accretion disk luminosity $L_{d}$. Right panel: distributions of the accretion disk luminosity in units of the Eddington one, black hole mass in units of solar mass, the ratio between $P_{r}$ and $L_{d}$, the ratio between the total jet power $P_{j}$ and $L_{d}$. Due to the two difference scenarios for $\gamma$-rays spectrum, the resultant distribution of the parameters are denoted in the legend of the corresponding panel.

the $\gamma$-ray emission, we can provide an acceptable fit to the $\mathrm{GeV} \gamma$ ray spectrum except for five sources 1Jy 1308, PKS 1502, 4C 01.28, PKS 0727 and S4 0917 truncated by $\mathrm{KN}$ effects. This implies that the spectral breaks observed in the Fermi-LAT spectra of many blazars can be well explained by a combination of two different Comptonscattered components for most sources. For the five sources, the $\gamma$ rays produced in the $\mathrm{KN}$ regime challenge the adopted model, opening the possibility of hadronic or leptohadronic origin of the emission (Böttcher et al., 2013; Dermer et al., 2014). For the other five sources PKS 0347, S3 2141, 4C 29.45, PKS 2325, and PKS 0528, the contribution of the ECC can be neglected, and the $\gamma$ ray spectrum may be represented either by the ECD or by ECT. It implies that the derived energy density $U_{B L R}$ is roughly at maximum, and the spectral break may be caused by the high-energy cutoff in the electron distribution suggested by Abdo et al. (2009). The results indicate that the SEDs modeling alone does not allow one to distinguish if the $\mathrm{GeV}$ emission of powerful blazars takes place within the sub-pc BLR or farther out within the pc DT, when the geometry structure of the external environment is poorly known.

At the last of the section, we calculated the jets power. The jet's kinetic power can be written in the sum of the form of magnetic field $P_{B}$, relativistic electrons $P_{e}$, cold protons $P_{p}$ and produced radiation $P_{r}$, i.e., $P_{j e t}=P_{B}+P_{e}+P_{p}+P_{r}$ in the 
stationary frame of the host galaxy, which are calculated as $P_{i}=\pi R_{b}^{\prime 2} \Gamma_{b}^{2} U_{i}^{\prime} c$, where $U_{i}^{\prime}$ are the energy densities associated with the emitting electrons $U_{e}^{\prime}$, magnetic field $U_{B}^{\prime}$, cold protons $U_{p}^{\prime}$ and produced radiation field $U_{r}^{\prime}$ in the co-moving frame, respectively. We calculate $U_{p}^{\prime}$ by assuming one proton per emitting electron, then $U_{p}^{\prime}=$ $\int m_{p} c^{2} n_{e}\left(\gamma^{\prime}\right) d \gamma^{\prime}=U_{e}^{\prime} m_{p} /\left\langle\gamma^{\prime}\right\rangle m_{e}$, where $\left\langle\gamma^{\prime}\right\rangle=\int \gamma^{\prime} n_{e}\left(\gamma^{\prime}\right) d \gamma^{\prime} / \int n_{e}\left(\gamma^{\prime}\right) d \gamma^{\prime}$ is the average Lorentz factor of the relativistic electrons. The power that the jet spends to produce the radiation, $P_{r}$, can be written as $P_{r}=L_{r} \Gamma_{b}^{2} / 4 \delta_{b}^{4}$, where $L_{r}=4 \pi d_{L}^{2} \int\left[\epsilon F_{s y n}(\epsilon)+\right.$ $\left.\epsilon F_{s s c}(\epsilon)+\epsilon F_{e c}(\epsilon)\right] d \ln \epsilon$ is the total observed non-thermal luminosity.

The black hole mass $M_{B H}$ and the disc luminosity in units of the Eddington one $L_{d} / L_{e d d}$, or, equivalently, the accretion rate, are two crucial parameters in understanding the evolution of the external environment in the nuclei of blazars (Böttcher \& Dermer , 2002; Ghisellini \& Tavecchio, 2008; Ghisellini et al., 2009; Elitzur, 2014). As the accretion rate decreases below a critical value, the BLR will disappear, or becomes weak and very close to the central $\mathrm{BH}$, and subsequently the equivalent width of emission lines is depressed, or the lines are entirely absent. The left panel of Fig. 4 compares the distribution of the jet power with that of the accretion luminosity. The accretion disc luminosity $L_{d}$ is in the range of $8 \times 10^{44} \sim$ $8 \times 10^{46} \mathrm{erg} / \mathrm{s}$. The distributions of $L_{d} / L_{e d d}, P_{r} / L_{d}$ and $P_{j} / L_{d}$ are shown in the right panel of Fig. 4, in which the distribution of the central BH mass $M_{B H} \sim(5-60) \times$ $10^{8} M_{\odot}$ derived through the SED modeling is also presented. Ghisellini \& Tavecchio (2015) compared the black hole mass estimated through the disc fitting method with the virial masses, and found that the masses derived by the two methods, on average, differ only slightly. Our results indicate that the power carried in radiation $P_{r}$ is roughly equivalent with $P_{e}$ or $P_{B}$, and more often a factor of $\sim 2-20$ times smaller than the accretion disc luminosity $L_{d}$. For most sources, the total jet power $P_{j}$ is a factor of $1-5$ greater than $L_{d}$. Ghisellini \& Tavecchio (2014) point out that the jet power as measured through the $\gamma$ ray luminosity dominates over the disk luminosity as measured by broad emission lines. Under the equipartition condition, we reach the conclusions that the jets of FSRQs are powerful and matter dominated and transforming a few per cent of their kinetic power into radiation (Ghisellini et al., 2010; Ghisellini \& Tavecchio, 2014).

\subsection{External radiation field and locations of the HE emission region}

The locations of emission regions are inferred from the covering factor of DT or ECD process (Dermer \& Schlickeiser, 1993; Dermer et al., 2009; Kushwaha et al., 2013; Cao \& Wang, 2013). In the lower right of Fig. 3, the distributions of the locations of the BLR and DT derived from the accretion disk luminosity $L_{d}$ are compared with those of the locations $Z_{l o c}$ estimated from two different prescriptions. It can be found that the HE emission take place in the range $\sim 500-2500 R_{g}$ determined by the Compton-scattered disk radiation. The results are in good agreement with previous works. The second scenario places the HE emission region at $\sim 10^{4}-2 \times 10^{5} R_{g}$ estimated from the covering factor of the DT. We can also find that the locations derived from SEDs modeling in model A are around the locations of the BLR, while on the other hand the locations of the emission region estimated from the covering factor are around the locations of the DT. In Model A the emission region of 13 out of 27 are outside of the BLR, while 18 out of 27 are outside the DT in the Model B. 
Plausible values of external radiation energy densities $\left(U_{B L R}\right.$ and $\left.U_{I R}\right)$ can be obtained by fitting the high-energy part of the observed SEDs. For most sources the derived energy density $U_{B L R}$ is comparable with the energy density $U_{D T}$. In Model B we adopted the same energy density $U_{B L R}$ in Model A for all sources in order to reproduce the $\gamma$ ray spectrum. Based on the SEDs modeling, we find that the derived values of the energy densities $U_{d}, U_{B L R}$ and $U_{D T}$ are restricted to the range of $\sim$ $0.03-3.73, \sim(0.1-9.0) \times 10^{-4}$, and $\sim(0.1-41.63) \times 10^{-4} \mathrm{erg} / \mathrm{cm}^{3}$, respectively, except for S3 2141 .

For S3 2141, the required energy density of external radiation field is either $14.79 \mathrm{erg} / \mathrm{cm}^{3}$ for the accretion disk, or $0.023 \mathrm{erg} / \mathrm{cm}^{3}$ for the DT, which are responsible for the Compton-scattered disk and DT component, respectively. For both cases, the Comptonscattered BLR radiation can be neglected. The location of the emission region estimated from the Compton-scattered disk is about $0.01 \mathrm{pc}$, which is in accord with the allowed minimum distance from the central engine (Ghisellini \& Madau, 1996). For this source, the energy density $U_{D T}$ corresponds to the hot dust $T \sim 1300 \mathrm{~K}$ at $0.3 \mathrm{pc}$ from the central engine.

From Table 1 we find that the dust temperatures of most sources are in the range $200-800 \mathrm{~K}$ and decrease with increasing distance from the central engine, if the HE emission regions take place from a few parsecs to tens of parsecs. It implies that the hotter emitting material is expected to be closer to the central engine. Previous works also suggest the presence of a warm dust with the temperature $150-800 \mathrm{~K}$ extending up to a few tens of parsec (Cleary et al., 2007; Nenkova et al., 2008; Landt et al., 2010; Malmrose et al., 2011).

The result indicates that the values of $U_{B L R}$ have to be compared with the values two orders of magnitude larger adopted by other authors (e.g., Ghisellini et al., 2010; Ghisellini \& Tavecchio, 2014). The values are derived from the observation on the assumption of a spherical structure and a typical value of a covering factor, and subsequently put constraint on the properties of the jets in those work. As mentioned in the Introduction, the geometry structure is not clear. In our work, $U_{B L R}$ is derived from a SED modeling, and is comparable to the result of Cao \& Wang (2013) and Böttcher et al. (2013), where the values are derived with a similar approach, and their work support the condition of equipartition. Such a low energy densities may be in favor of a disk-like geometry, at least a aperture angle $<90^{\circ}$, if a emission region sites in the BLR. Actually, with the decrease of the aperture angle, the illuminating continuum intercepted by the BLR clouds decreases, leading to the decrease of the flux of the reprocessed emissions and the diffuse continuum (Lei \& Wang, 2014). The lack of a rigorous $\chi^{2}$ minimization strategy prevents us to further constrain the geometry and extend of BLR.

\section{ACKNOWLEDGMENT}

We thank the anonymous referee for constructive comments and suggestions which significantly improved the presentation of our results. We acknowledge Dr. Dahai Yan for helpful discussion. This work is supported partially by the National Science Foundation of China (grants U1531131, 11433004), and the Top Talents Program of 
Yunnan Province, China.

\section{References}

Abdo A. A. et al., 2009, ApJ, 699, 817

Abdo, A. A., et al. 2010, ApJ, 716, 30

Ackermann, M., et al., 2012, Sci, 338, 1190

Agudo, I., et al., 2011, ApJ, 726, L13

Bloom, S. D., \& Marscher, A. P. 1996, ApJ, 461, 657

Blazejowski, M., et al., 2000, ApJ, 545, 107

Blumenthal, G. R., \& Gould, R. J., 1970, Rev. Mod. Phys., 42, 237

Böttcher, M., \& Dermer, C. D. 2002, ApJ, 564, 86

Böttcher, M., \& Chiang, J., 2002, ApJ, 581, 127

Böttcher, M., Reimer, A., Sweeney, K., Prakash, A., 2013, ApJ, 768, 54

Gao, X. Y., Wang, J. C., Zhou, M., 2011, RAA, 11, 902

Cao, G., Wang, J. C., 2013, MNRAS, 436, 2170

Castignani, G., Haardt, F., Lapi, A., et al., 2013, A\&A, 560, 28

Castignani, G., \& De Zotti, G., 2015, A\&A, 573A, 125

Celotti A., \& Ghisellini G. 2008, MNRAS, 385, 283

Cerruti, M., Dermer, C. D., et al., 2013, ApJ, 771, 4

Cleary, K., Lawrence, C. R., Marshall, J. A., Hao, L., Meier, D., 2007, ApJ, 660, 117

Crusius, A., \& Schlickeiser, R., 1986, A\&A, 164, L16

Dermer, C. D., Sturner, S. J. \& Schlickeiser, R., 1997, ApJ, 109, 103

Dermer, C. D. \& Schlickeiser, R., 1993, ApJ, 416, 458

Dermer, C. D. \& Schlickeiser, R., 2002, ApJ, 575, 667

Dermer, C. D. \& Atoyan, A., 2004, ApJ, 611, L9

Dermer, C. D., et al., 2009, ApJ, 692, 32

Dermer, C. D., Cerruti, M., et al., 2014, ApJ, 782, 82

Decarli, R., Labita, M., Treves, A., \& Falomo, R., 2008, MNRAS, 387, 1237 
Decarli, R., Dotti, M., \& Treves, A., 2011, MNRAS, 413, 39

Dotson, A., Georganopoulos, M., et al., 2012, ApJ, 758, L15

Elitzur, M., Ho, L. C. \& Trump, J. R., 2014, MNRAS 438, 3340

Fanaroff, B. L. \& Riley, J. M. 1974, MNRAS, 167, 31

Finke, J. D., Dermer, C. D. \& Böttcher, M., 2008, ApJ, 686, 181

Finke, J. D., Razzaque, S., \& Dermer, C. D. 2010, ApJ, 712, 238

Finke, J. D., Dermer, C. D., 2010, ApJ, 714, 303

Gould, R. J., Schréder, G. P. 1967, Phys.Rev., 155, 1408

Ghisellini G., \& Madau P., 1996, MNRAS, 280, 67

Ghisellini, G., Foschini, L., Tavecchio, F. \& Pian, E., 2007, MNRAS, 382, 82

Ghisellini, G., Maraschi, L. \& Tavecchio, F., 2009, MNRAS, 396, 105

Ghisellini, G. \& Tavecchio, F., 2008, MNRAS, 387, 1669

Ghisellini, G. \& Tavecchio, F., 2009, MNRAS, 397, 985

Ghisellini, G., Tavecchio, F., Ghirlanda, G., et al., 2010, MNRAS, 402, 497

Ghisellini, G., Tagliaferri, G., \& Foschini, L. et al. 2011, MNRAS, 411, 901

Ghisellini, G., 2013, LNP, 873

Ghisellini, G., \& Tavecchio, F., 2014, Natur, 515, 376

Ghisellini, G., \& Tavecchio, F., 2015, MNRAS, 448, 1060

Giommi, P., et al., 2012, A\&A 541, 160

Harris, J., Daniel, M. K., Chadwick, P. M., 2012, ApJ, 761, 2

Hu, W., Fan, Z. H., \& Dai, B. Z., 2015, RAA, 15, 1455

Jorstad, S. G. et al., 2005, AJ, 130, 1418

Jarvis, M. J., \& McLure, R. J., 2006, MNRAS, 369, 182

Katarzyński, K., 2012, A\&A, 537, 47

Kang, Shiju, Chen, Liang, Wu, Qingwen, 2014, ApJS, 215, 5

Kushwaha, P., Sahayanathan, S., \& Singh, K. P., 2013, MNRAS, 433, 2380

Lei, Maichang \& Wang, Jiancheng, 2014, PASJ, 66, 7

León-Tavares, J., Lobanov, A. P., Chavushyan, V. H., et al. 2010, ApJ, 715, 355 
León-Tavares, J., et al., 2013, ApJ, 763, 36

Landt, H., Buchanan, C. L., \& Barmby, P., 2010, MNRAS, 408, 1982

Liu, H. T., Bai, J. M., \& Wang, J. M. 2011, MNRAS, 414, 155

Ludovic, S. \& Gilles, H., 2004, ApJ, 616, 136

Labita, M., Treves, A., Falomo, R., \& Uslenghi, M., 2006, MNRAS, 373, 551

Maraschi, L., Ghisellini, G., \& Celotti, A., 1992, ApJ, 397, L5

Moderski, R., Sikora, M., \& BŁażejowski, M., 2003, A\&A, 406, 855

Malmrose, M. P., Marscher, A. P., Jorstad, S. G., Nikutta, R., \& Elitzur, M., 2011, ApJ, 732,116

Marscher, A. P. et al. 2008, Nature, 452, 966

Marscher, A. P., Jorstad, S. G., Larionov, V. M., et al. 2010, ApJ, 710, L126

Nalewajko, K., Begelman, M. C., Sikora, M. 2014, ApJ, 789, 161

Nikishov, A. I. 1961, Zh. Experimen. i Theo. Fiz., 41, 549

Nenkova, M., Sirocky, M. M., et al., 2008, ApJ, 685, 160

Orienti, M., et al., 2013, MNRAS, 428, 2418

Peterson, B. M. 1993, PASP, 105, 247

Peterson, B. M., et al. 2004, ApJ, 613, 682

Shakura, N. I., \& Sunyaev, R. A., 1973, A\&A, 24, 337

Schlickeiser, R., \& Lerche, I., 2007, A\&A, 476, 1

Schlickeiser, R., \& Lerche, I., 2008, A\&A, 485, 315

Sikora, M., Begelman, M. C., \& Rees, M. J. 1994, ApJ, 421, 153

BŁażejowski, M., Sikora, M., et al., 2000, ApJ, 545, 107

Sikora, M., Moderski, R., \& Madejski, G. M., et al., 2008, ApJ, 675, 71

Savolainen, T., Homan, D. C., et al., 2010, A\&A, 512, 24

Sanchez, D. A., et al., 2015, arXiv: 150600965S

Tavecchio, F., Maraschi, L., \& Ghisellini., G., 1998, ApJ, 509, 608

Tavecchio, F., \& Ghisellini, G., 2008, MNRAS, 386, 945

Tavecchio, F., Ghisellini, G., Bonnoli G., Ghirlanda, G., 2010, MNRAS, 405, 94 
Tang, Y. Y., Dai, Z. C, Zhang, L., 2010, RAA, 10, 415

Urry, C. M. \& Padovani, P. 1995, PASP, 107, 803

Vlahakis, N., Königl, A. 2004, ApJ, 605, 656

Yan, D. H., Zeng, H. D., Zhang, L., 2012, PASJ, 64, 80

Yan, D. H., Fan, Z. H., Zhou, Y., Dai, B. Z., 2013, RAA, 13, 411

Yan, D. H., Zeng, H. D., Zhang, L., 2014, MNRAS, 439, 2933 


\section{Appendix A. Constraints from quasi-simultaneous MWL SEDs}

In this appendix, the relations between observable quantities and the physical parameters of the model are derived on the base of a commonly accepted synchrotron radiation and SSC emission of the same relativistic electrons. We neglect the modification due to the $\mathrm{KN}$ reduction, which is more important for the high-synchrotron peaked BL Lac objects (Tavecchi et al., 1998).

The normalization factor $k_{e}^{\prime}$ of the relativistic electrons spectra is determined by equipartition parameter $\xi_{e}$, which is the fraction of the magnetic field energy density $U_{B}^{\prime}$. As a consequence of this parameterization, the electron energy density can be calculated by

$$
u_{e}^{\prime}=\xi_{e} U_{B}^{\prime}=m_{e} c^{2} \int_{\gamma_{\min }^{\prime}}^{\gamma_{\max }^{\prime}} \gamma^{\prime} n_{e}^{\prime}\left(\gamma^{\prime}\right) d \gamma^{\prime} .
$$

Substituting Eq.1 into above equation, we obtain the following expression for $k_{e}^{\prime}$ :

$$
k_{e}^{\prime}=\frac{\xi_{e} U_{B}^{\prime}}{\gamma_{b r}^{\prime}{ }^{2} m_{e} c^{2} f_{1}},
$$

where $f_{1}=g_{10}+g_{11}$, and

$$
\begin{aligned}
& g_{10}=\left\{\begin{array}{ll}
\ln \left(\gamma_{b r}^{\prime} / \gamma_{\text {min }}^{\prime}\right) & p_{1}=2 \\
{\left[1-\left(\gamma_{\min }^{\prime} / \gamma_{b r}^{\prime}\right)^{2-p_{1}}\right] /\left(2-p_{1}\right)} & p_{1} \neq 2
\end{array},\right. \\
& g_{11}=\left\{\begin{array}{ll}
\ln \left(\gamma_{\max }^{\prime} / \gamma_{b r}^{\prime}\right) & p_{2}=2 \\
{\left[\left(\gamma_{\max }^{\prime} / \gamma_{b r}^{\prime}\right)^{2-p_{2}}-1\right] /\left(2-p_{2}\right)} & p_{2} \neq 2
\end{array} .\right.
\end{aligned}
$$

The spectral indexes below and above the break energy $\gamma_{b r}^{\prime} m_{e} c^{2}$ are determined from the observed SEDs. For the synchrotron and the up-scattered synchrotron spectrum in the Thomson limit, the energy spectral index $\alpha$ is related to the electron slope $p$ according to the relationship, $\alpha=(p-1) / 2$ (Blumenthal \& Gould, 1970; Dermer \& Schlickeiser, 1993; Dermer \& Sturner, 1997; Dermer \& Schlickeiser, 2002; Tavecchi et al., 1998; Katarzyński, 2012; Moderski et al., 2003; BŁażejowski et al., 2000; Sikora et al., 2008). But we note that the relation becomes invalid when $p<1 / 3$ for synchrotron emission. In such a case the spectral index of the power law part of the synchrotron emission is constant, and $\alpha=-1 / 3$, which is equivalent to the spectral index of the synchrotron emissivity of a mono-energetic population of the electrons. The spectral index of the SSC emission is also limited by the value $\alpha=-1 / 3$. The $\nu F_{\nu}$ spectral energy index $\alpha_{\text {sed }}$ of the SSC and EC scenarios are $-4 / 3$ and -2 , respectively, below the minimum up-scattered photon energy corresponding to the minimum Lorentz factor $\gamma_{\text {min }}^{\prime}$.

In our approach, the parameters $\gamma_{\min }^{\prime}$ and $\gamma_{\max }^{\prime}$ enter as free parameters, but their values are reasonably constrained by the conditions posed by the SEDs. The lowenergy tail of the EC component can pose severe constraints on the value of $\gamma_{\min }^{\prime}$ (Ghisellini et al., 2007; Celotti \& Ghisellini, 2008), while the value of $\gamma_{\max }^{\prime}$ can be constrained by the HE end of the $\gamma$-ray spectrum and/or the decreasing part of the Optical/UV band. For the luminous blazars, the photons at $\nu_{s y}^{p k}$ dominate the energy 
density of the target photons, meanwhile the inverse Compton power in the Thomson regime will be dominated by photons at frequency $\nu_{s s c}^{p k}=(4 / 3) \gamma_{b r}^{\prime}{ }^{2} \nu_{s y}^{p k}$. Inverting the equation, the break Lorentz factor is approximately given by

$$
\gamma_{b r}^{\prime}=\sqrt{\frac{3 \nu_{s s c}^{p k}}{4 \nu_{s y}^{p k}}} .
$$

The total synchrotron luminosity, neglecting the self-absorption correction, is

$$
L_{\text {syn }}=m_{e} c^{2} V_{b}^{\prime} \delta_{b}^{4} \int_{1}^{\infty}\left|\dot{\gamma}_{s y n}^{\prime}\right| n_{e}^{\prime}\left(\gamma^{\prime}\right) d \gamma^{\prime},
$$

where $\dot{\gamma}_{s y n}^{\prime}=-4 c \sigma_{T} U_{B}^{\prime} \gamma^{\prime 2} / 3 m_{e} c^{2}$ is the synchrotron cooling rate. Then, the total synchrotron luminosity can be calculated by

$$
L_{s y n}=\frac{4}{3} c \sigma_{T} U_{B}^{\prime} V_{b}^{\prime} \delta_{b}^{4} k_{e}^{\prime} \gamma_{b r}^{\prime 3} f_{2}=\frac{f_{2}}{f_{1}} \frac{4 c \sigma_{T}}{3 m_{e} c^{2}} \gamma_{b r}^{\prime} V_{b}^{\prime} \xi_{e} U_{B}^{\prime 2} \delta_{b}^{4}
$$

where $f_{2}=g_{20}+g_{21}$, and

$$
\begin{aligned}
& g_{20}=\left\{\begin{array}{ll}
\ln \left(\gamma_{b r}^{\prime} / \gamma_{\text {min }}^{\prime}\right) & p_{1}=3 \\
{\left[1-\left(\gamma_{\text {min }}^{\prime} / \gamma_{b r}^{\prime}\right)^{3-p_{1}}\right] /\left(3-p_{1}\right)} & p_{1} \neq 3
\end{array},\right. \\
& g_{21}=\left\{\begin{array}{ll}
\ln \left(\gamma_{\max }^{\prime} / \gamma_{b r}^{\prime}\right) & p_{2}=3 \\
{\left[\left(\gamma_{\max }^{\prime} / \gamma_{b r}^{\prime}\right)^{3-p_{2}}-1\right] /\left(3-p_{2}\right)} & p_{2} \neq 3
\end{array} .\right.
\end{aligned}
$$

The averaged synchrotron frequency for an electron of Lorentz factor $\gamma^{\prime}$ is $\epsilon^{\prime}=(4 / 3) \gamma^{\prime 2} \epsilon_{B}^{\prime}$. Using a $\delta$-function approximation for the averaged synchrotron frequency, the synchrotron luminosity at a specific frequency is written as,

$$
\epsilon^{\prime} L_{\text {syn }}^{\prime}\left(\epsilon^{\prime}\right)=\left|\dot{\gamma}_{\text {syn }}^{\prime}\right| m_{e} c^{2} V_{b}^{\prime} \gamma^{\prime} n_{e}^{\prime}\left(\gamma^{\prime}\right)\left[d \ln \gamma^{\prime} / d \ln \epsilon^{\prime}\right],
$$

and reaches its maximum at the dimensionless peak frequency

$$
\epsilon_{s y}^{p k}=(4 / 3)\left[B_{c r}(1+z)\right]^{-1} \gamma_{b r}^{\prime 2} B^{\prime} \delta_{b} .
$$

The peak luminosity of the $\nu L_{\nu}$ synchrotron spectrum is straightforward to derive

$$
L_{s y}^{p k}=\frac{2}{3} c \sigma_{T} U_{B}^{\prime} \gamma_{b r}^{\prime}{ }^{3} V_{b}^{\prime} k_{e}^{\prime} \delta^{4}=\frac{2 c \sigma_{T}}{3 m_{e} c^{2} f_{1}} \gamma_{b r}^{\prime} V_{b}^{\prime} \xi_{e}{U_{B}^{\prime}}^{2} \delta^{4} .
$$

Inverting the above equation and using Eq.A.11, one obtains the intrinsic volume of the emitting region,

$$
V_{b}^{\prime}=\frac{L_{s y}^{p k}}{\left[(1+z) \epsilon_{s y}^{p k}\right]^{4}} \frac{f_{1} \gamma_{b r}^{\prime 7}}{\xi_{e}}\left(\frac{4}{3}\right)^{4} \frac{46 \pi^{2} m_{e} c^{2}}{c \sigma_{T} B_{c r}^{4}} .
$$

In a blob geometry, the volume is $V_{b}^{\prime}=4 \pi R_{b}^{\prime 3} / 3$. Then the dimension of the blob is given by,

$$
R_{b}^{\prime} \simeq 2.62 \times 10^{15} \xi_{e}^{-1 / 3} f_{1}^{1 / 3} \gamma_{100}^{\prime 7 / 3} L_{48}^{1 / 3}\left[(1+z) \nu_{13}\right]^{-4 / 3} .
$$


Another relation can be obtained from the SSC-dominated factor, $q$, which is directly related to the ratio between the synchrotron radiation and the magnetic field energy densities inside the blob. In the Thomson regime, $q=L_{s s c} / L_{s y n}=u_{s y n}^{\prime} / U_{B}^{\prime}=$ $\xi_{s}$, where the co-moving optically thin synchrotron energy density $u_{\text {syn }}^{\prime}=L_{s y n} R_{b}^{\prime} / V_{b} c \delta_{b}^{4}$, $L_{s s c}$ and $L_{s y n}$ are the observed SSC and synchrotron luminosities, respectively. Using Eq. (A.7) for $L_{s y n}$, we derive

$$
\xi_{s}=\frac{u_{s y n}^{\prime}}{U_{B}^{\prime}}=\frac{f_{2}}{f_{1}} \frac{4 \sigma_{T} R_{b}^{\prime}}{3 m_{e} c^{2}} \gamma_{b r}^{\prime} \xi_{e} U_{B}^{\prime} .
$$

For our aims it is useful to express the total luminosity through the peak luminosity in the $\nu L_{\nu}$ versus $\nu$ diagram, which is more accessible to observations. By comparing of Eq. (A.7) with Eq. (A.12), we see that the relationship between $L_{s y n}$ and $\nu L_{\nu}$ peak synchrotron luminosity is $L_{s y n}=2 f_{2} L_{s y}^{p k}$. The ratio between the internal synchrotron and magnetic field energy densities is approximately estimated by $\xi_{s} \simeq L_{s s c}^{p k} / L_{s y}^{p k}$.

Inverting Eq. (A.15) to get an expression for magnetic field $B^{\prime}$, and using Eq. (A.14) for $R_{b}^{\prime}$ gives

$$
B^{\prime} \simeq 9.41 \xi_{e}^{-1 / 3} \xi_{s}^{1 / 2} f_{1}^{1 / 3} f_{2}^{-1 / 2} \gamma_{100}^{\prime-5 / 3} L_{48}^{-1 / 6}\left[(1+z) \nu_{13}\right]^{2 / 3} .
$$

Replacing this result in Eq. (A.11) gives

$$
\delta_{b} \simeq 28.48 \xi_{e}^{1 / 3} \xi_{s}^{-1 / 2} f_{1}^{-1 / 3} f_{2}^{1 / 2} \gamma_{100}^{\prime-1 / 3} L_{48}^{1 / 6}\left[(1+z) \nu_{13}\right]^{1 / 3} .
$$

Here $\nu_{13}$ is the synchrotron peak frequency in units of $10^{13} \mathrm{~Hz}, L_{48}$ is the synchrotron peak luminosity in units of $10^{48} \mathrm{erg} / \mathrm{s}, \gamma_{100}^{\prime}$ is the break Lorentz factor in units of 100 .

\section{Appendix B. Spectral Energy Distributions}



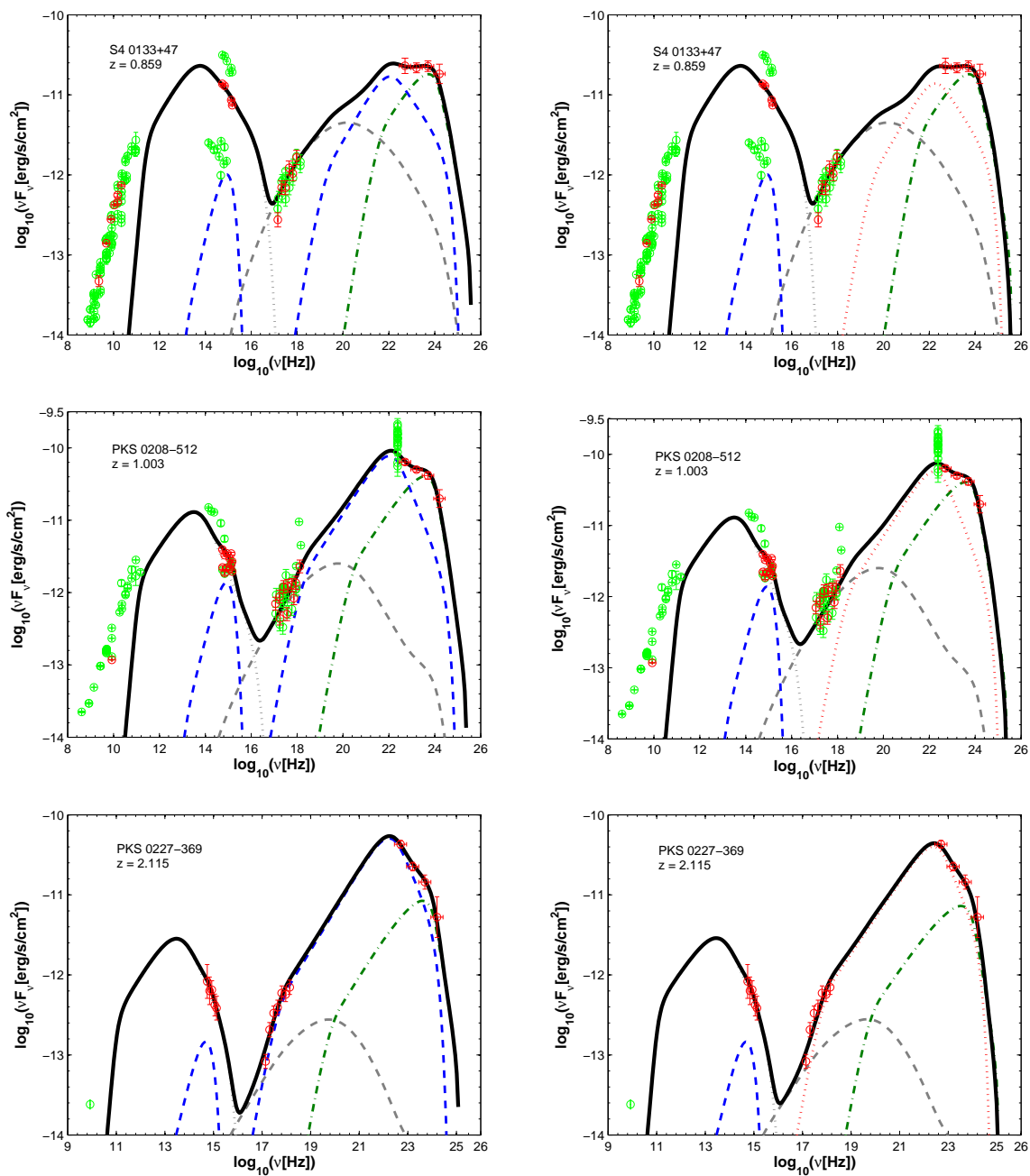

Figure B.5: Comparisons of the modelling SEDs with the observed data. For each source, the grey dotted, grey dashed and green dotted-dashed lines are the synchrotron, SSC and ECC, respectively, the blue dashed line is the thermal emission from the accretion disc and the ECD radiation, the red dotted line represents the ERC radiation, the thick solid line is the sum of the all components. The quasi-simultaneous MWL data are indicated by open circle (red in the electronic version) while archival data are in green. 

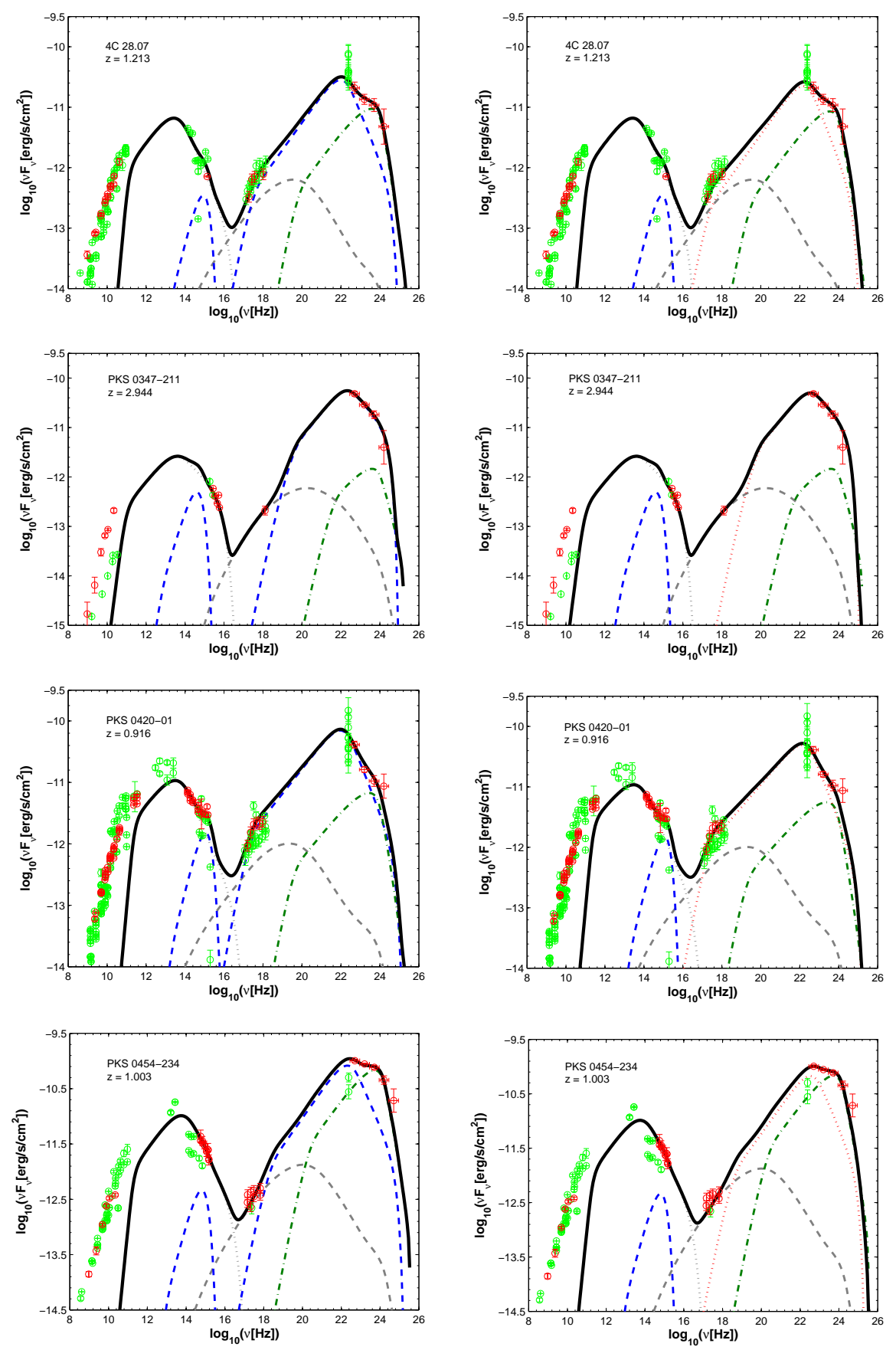

Fig. B.5 - continued. 

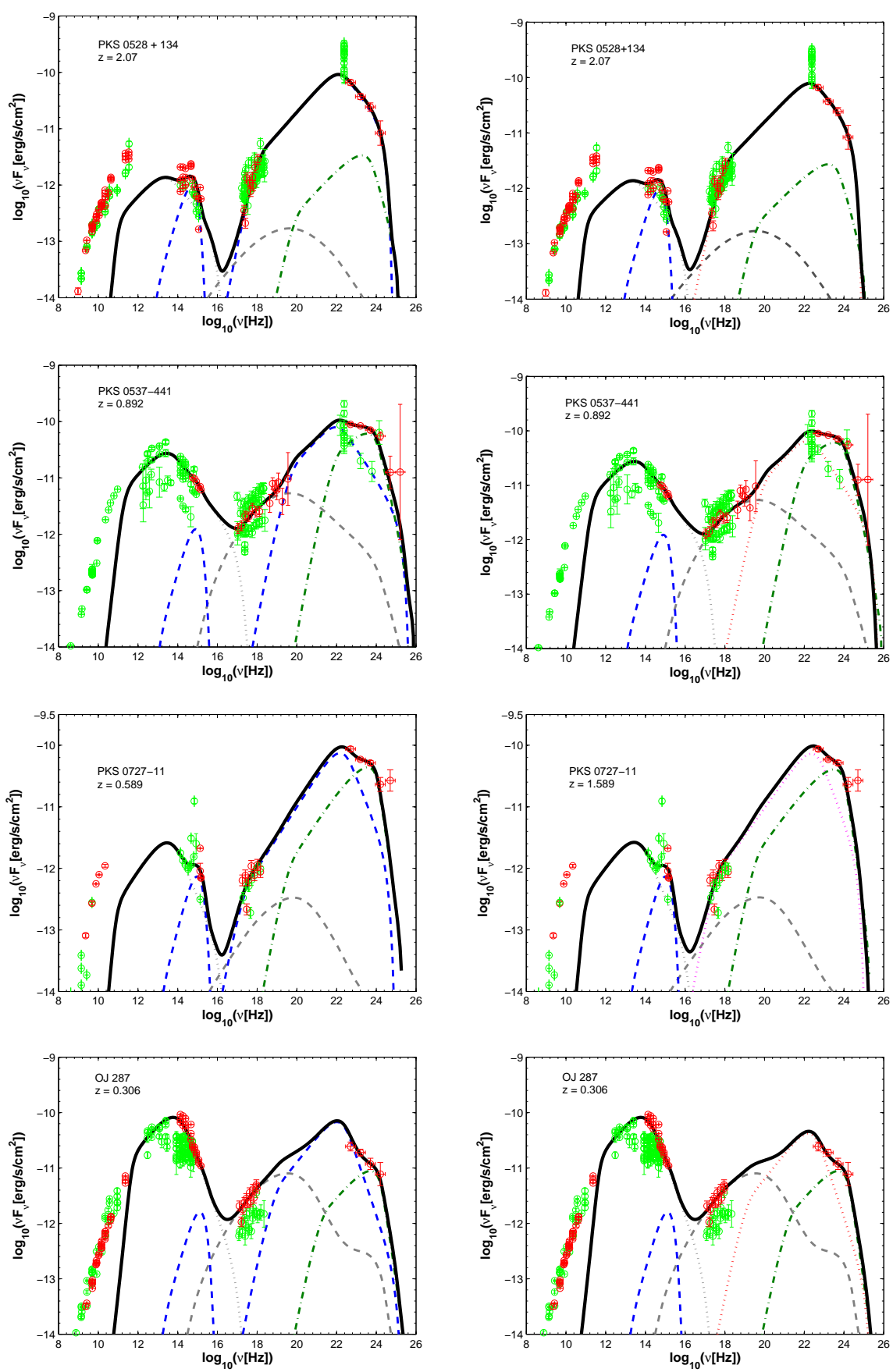

Fig. B.5 - continued. 

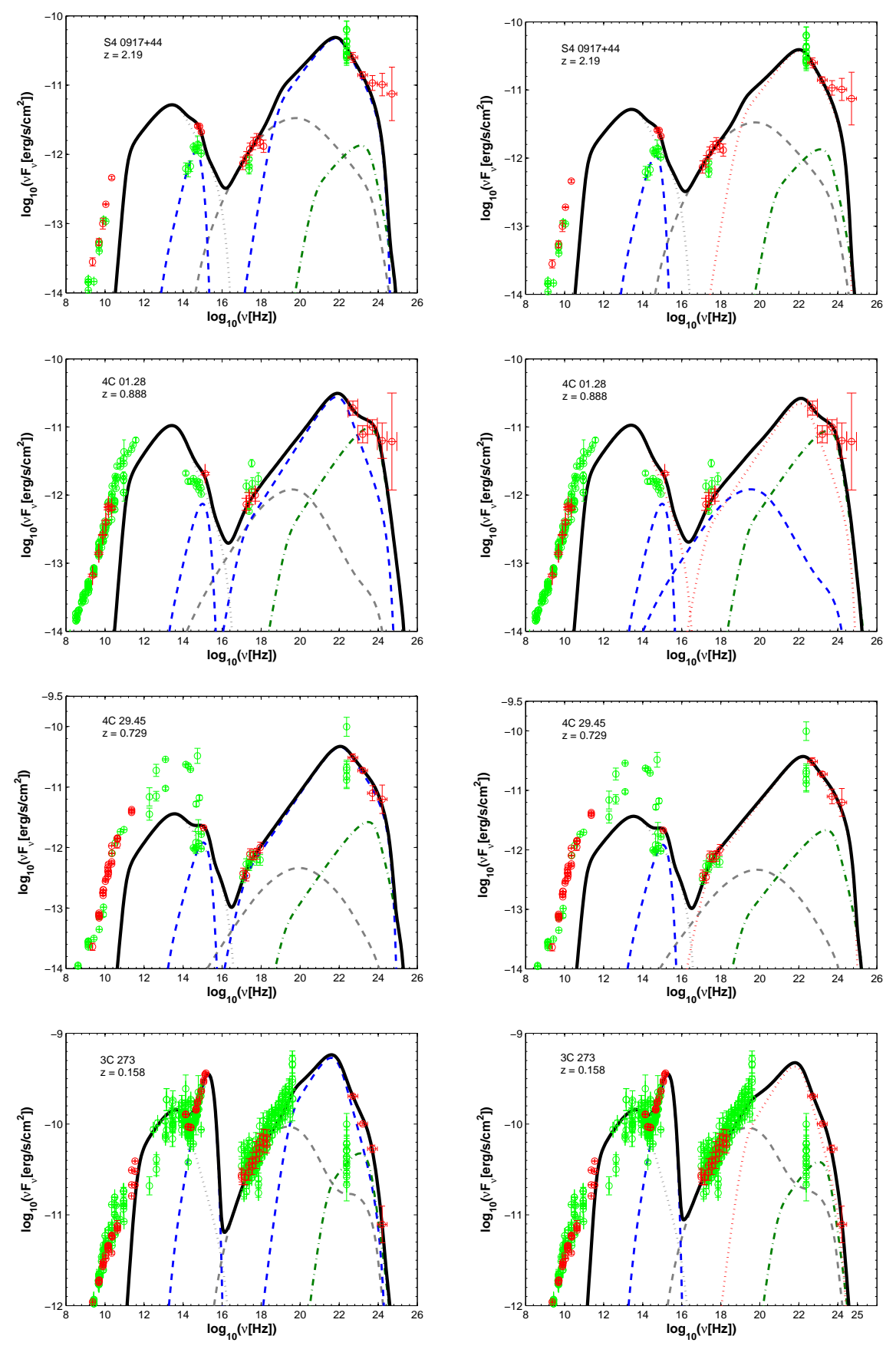

Fig. B.5 - continued. 

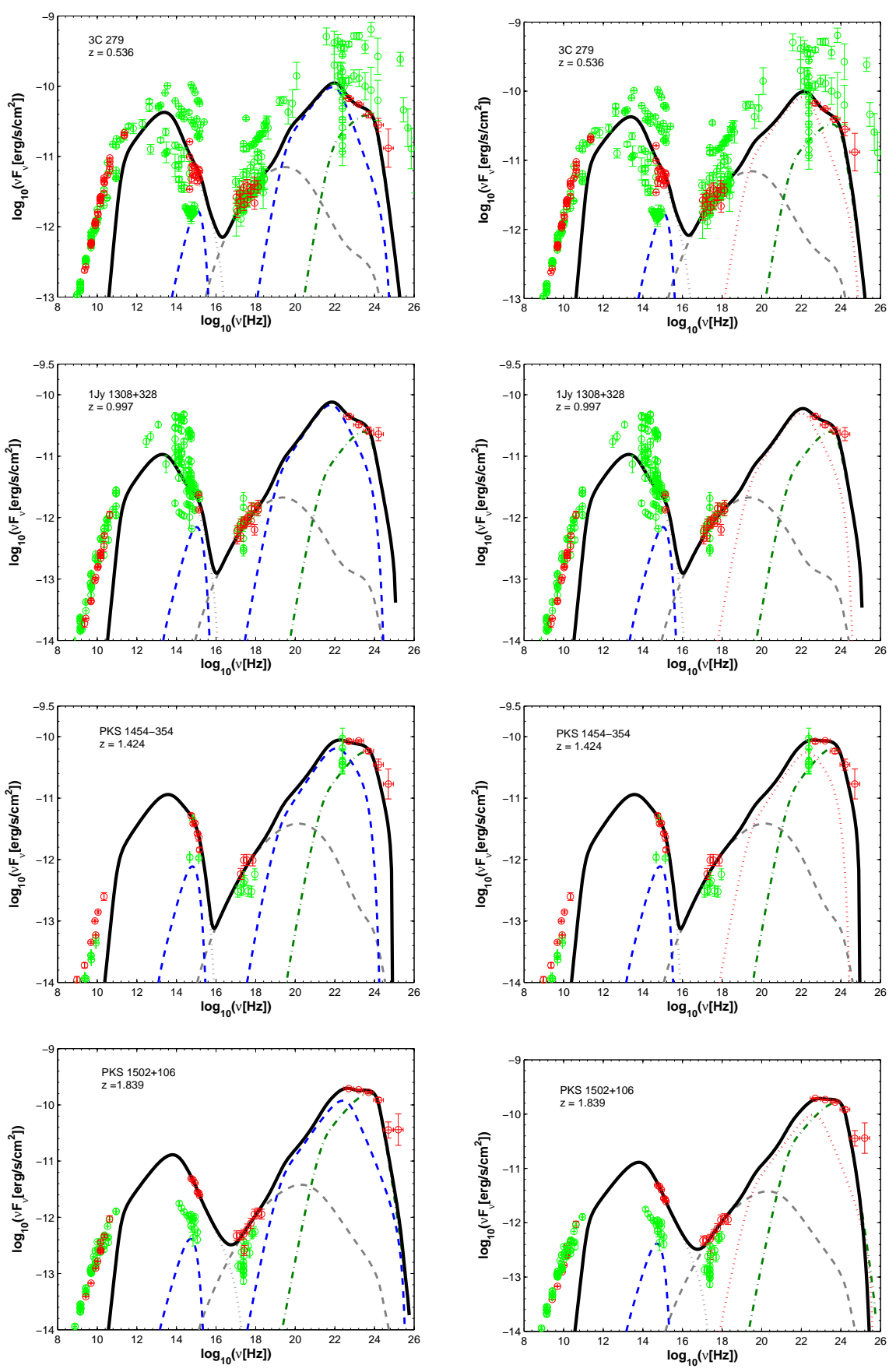

Fig. B.5 - continued. 

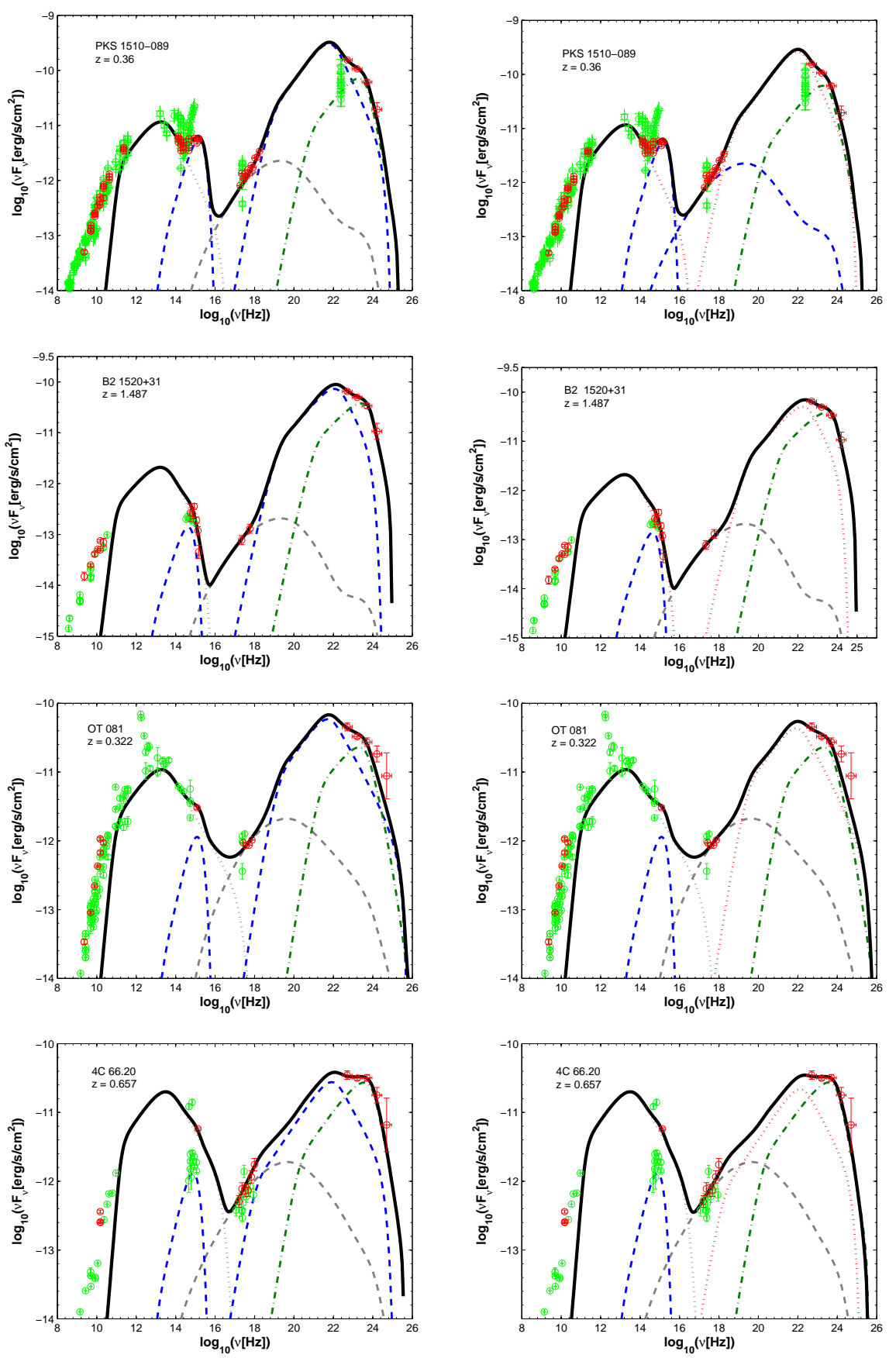

Fig. B.5 - continued. 

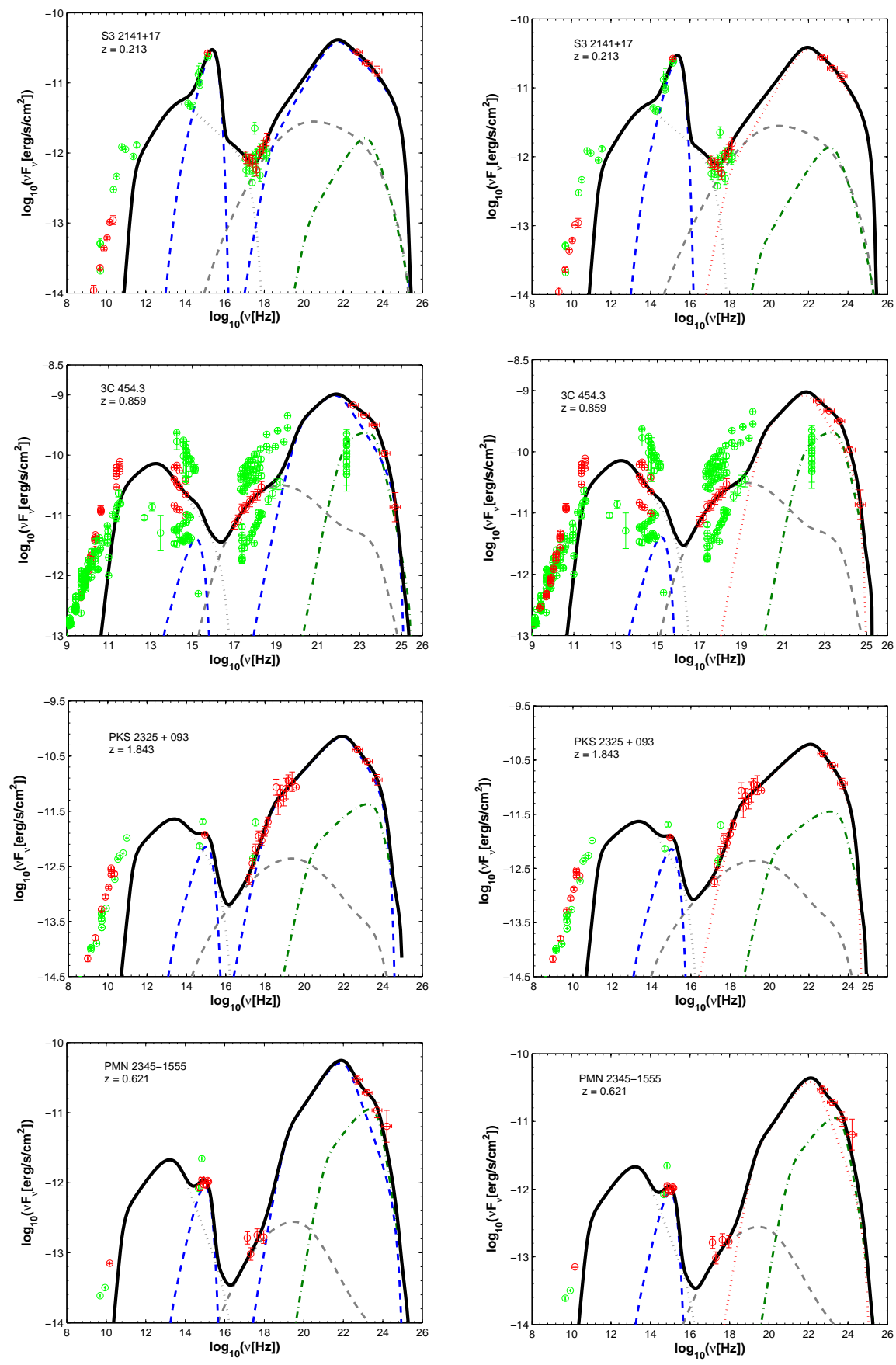

Fig. B.5 - continued. 